106. Flaegstad, T. et al. A possible contributory role of BK virus infection in neuroblastoma development. Cancer Res. 59, 1160-1163 (1999).

107. Lehtinen, M et al. Maternal herpesvirus infections and risk of acute lymphoblastic leukemia in the offspring. Am. J. Epidemiol. 158, 207-213 (2003).

108. Rivoltini, L. et al. Phenotypic and functional analysis of lymphocytes infiltrating paediatric tumours, with a characterization of the tumour phenotype. Cancer Immunol. Immunother. 34, 241-251 (1992).

109. Kataoka, Y., Matsumura, T., Yamamoto, S., Sugimoto, T. \& Sawada, T. Distinct cytotoxicity against neuroblastoma cells of peripheral blood and tumor-infiltrating lymphocytes from patients with neuroblastoma. Cancer Lett. 73, 11-21 (1993).

110. Facchetti, P. et al. Functional and molecular characterization of tumour-infiltrating lymphocytes and clones thereof from a major-histocompatibility-complexnegative human tumour: neuroblastoma. Cancer Immunol. Immunother. 42, 170-178 (1996).

111. Martin, R. F. \& Beckwith, J. B. Lymphoid infiltrates in neuroblastomas: their occurrence and prognostic significance. J. Pediatr. Surg. 3, 161-164 (1968).

112. Lauder, I. \& Aherne, W. The significance of lymphocytic infiltration in neuroblastoma. Br. J. Cancer 26, 321-330 (1972)

113. Dickens, D. S., Kozielski, R., Leavey, P. J., Timmons, C \& Cripe, T. P. Cyclooxygenase-2 expression does not correlate with outcome in osteosarcoma or rhabdomyosarcoma. J. Pediatr. Hematol. Oncol. 25 282-285 (2003).

114. Wiemels, J. L., Ford, A. M., Van Wering, E. R. Postma, A \& Greaves, M. Protracted and variable latency of acute lymphoblastic leukemia after TEL-AML1 gene fusion in utero. Blood 94, 1057-1062 (1999).

115. Wiemels, J. L. et al. In utero origin of $t(8 ; 21)$ AML1-ETO translocations in childhood acute myeloid leukemia. Blood 99, 3801-3805 (2002).
116. Borrello, I. \& Pardoll, D. GM-CSF-based cellular vaccines: a review of the clinical experience. Cytokine Growth Factor Rev. 13, 185-193 (2002).

117. Pardoll, D. M. Paracrine cytokine adjuvants in cancer immunotherapy. Annu. Rev. Immunol. 13, 399-415 (1995).

118. Buttiglieri, S., Galetto, A. Forno, S., De Andrea, M. \& Matera, L. Influence of drug-induced apoptotic death on processing and presentation of tumor antigens by dendritic cells. Int. J. Cancer 106, 516-520 (2003).

119. Ohndorf, U. M., Rould, M. A., He, Q., Pabo, C. O. 8 Lippard, S. J. Basis for recognition of cisplatin-modified DNA by high-mobility-group proteins. Nature $\mathbf{3 9 9}$, 708-712 (1999).

Acknowledgements

J.V. has received support from the Nona and Kullervo Väre Foundation, Finland, and the Finnish Cultural Foundation. M.T.L. has received grant support from the National Institutes of Health, United States.

Competing interests statement

The authors declare no competing financial interests.

\section{(1) Online links}

\section{DATABASES}

The following terms in this article are linked online to: Entrez Gene:

http://www.ncbi.nlm.nih.gov/entrez/query.fcgi?db=gene arginase | COX1 | COX2 | C-reactive protein | HMGB1 | IDO | IFN- $\alpha$ | IFN- $\gamma \mid$ IL-6 | IL-12 | IL-23 | LETAL | MICA | MICB | nitric-oxide synthase | NKG2D | RAGE | STAT3 | TNF

SUPPLEMENTARY INFORMATION

See online article: S1 (table)

Access to this interactive links box is available online.

\title{
Seventeen-colour flow cytometry: unravelling the immune system
}

\section{Stephen P. Perfetto, Pratip K. Chattopadhyay and Mario Roederer}

The increasing need for polychromatic approaches to flow cytometry, coupled with rapid technological advances, has pushed the frontiers of flow cytometry beyond 12-colour systems. Recent breakthroughs have allowed the design and implementation of instruments that measure 19 parameters (17 fluorescent colours and 2 physical parameters). This article describes the instrumentation and considers the reagents, analysis and applications for this powerful, new extension of flow-cytometric technology.

Fluorescence-activated flow cytometry is one of the most powerful technologies that is routinely used in immunology. It allows not only for the rapid multiparametric analysis of cells on a cell-by-cell basis, but also for the viable separation (sorting) of highly purified populations of cells. Flow cytometry was born in the Herzenberg laboratory at
Stanford University (Stanford, United States) in the late $1960 \mathrm{~s}^{1-3}$. Early cytometers measured three parameters (one fluorescence signal and two scattered-light signals); the power of multiparametric analysis to discriminate functionally distinct subsets of leukocytes drove the technology to the commonly used four-colour machines that can distinguish six parameters for each cell. This four-colour analysis was first performed in the mid-1980s. In the 1990s, further technological advances were made necessary by the growing appreciation of researchers for the complexity of the immune system; a systematic series of developments of hardware, software and chemistry were spearheaded by the Herzenberg laboratory to create machines capable of measuring more than ten colours ${ }^{4}$.

This technology has since revealed that there are hundreds of phenotypically distinct cell types in the peripheral blood of humans ${ }^{5}$.
The ability to discriminate between these cell types is crucial to our understanding of cellular immunity and disease pathogenesis. This can be illustrated by recent studies of HIV, which show considerable changes to specific T-cell subpopulations beyond 'bulk' $\mathrm{CD}^{+}{ }^{+} \mathrm{T}$ cells. For example, disease caused by HIV has been associated with a depletion of naive $e^{6,7}$ and newly produced ${ }^{8} \mathrm{~T}$ cells, viral infection of memory ${ }^{9}$ and HIV-specific ${ }^{10}$ $\mathrm{CD}^{+} \mathrm{T}$ cells, and clonal expansion of activated ${ }^{11}$ and $\mathrm{CD}^{+}$(REF. 12) T-cell subsets. These are specialized subsets of $\mathrm{T}$ cells that carry out distinct programmed functions; the selective change in their representation reflects a marked alteration in the composition of the immune system, even when overall numbers of $\mathrm{T}$ cells might not be significantly altered. For each of these studies, three- or four-colour flow cytometry techniques were sufficient. However, future studies will require the simultaneous measurement of more phenotypic and functional parameters. Technology that can measure six or more colours - polychromatic flow cytometry - is well suited to such endeavours; however, investigators need to determine how many colours are required to answer immunological questions.

For simple discrimination between peripheral blood cells, as few as two physical parameters (forward and side scatter) and two fluorescence parameters are sufficient. Forward- and side-scatter characteristics can be used to distinguish monocytes and granulocytes (high side scatter and high forward scatter) from platelets and erythrocytes (low side scatter and low forward scatter). Lymphocytes and circulating dendritic cells show intermediate forward and side scatter and can be further segregated by lineage (that is, B cells, T cells or natural killer (NK) cells) using one or more fluorescence parameters. For example, T cells can be identified using fluorescently labelled antibodies specific for the CD3 cell-surface marker and either the CD4 or CD8 cell-surface markers (two-colour flow cytometry).

Within T-cell populations, various markers have been proposed to distinguish naive, effector- and memory-cell populations. These markers are often paired (for example, CD45RA and CD62 ligand (CD62L), coexpression of which identifies naive $\mathrm{T}$ cells) to create the simple phenotyping schemes commonly used in four-colour experiments. However, attempts to describe the complex T-cell compartment using these phenotyping schemes have raised many questions. For example, most of the common markers that are used to identify the stages of T-cell 


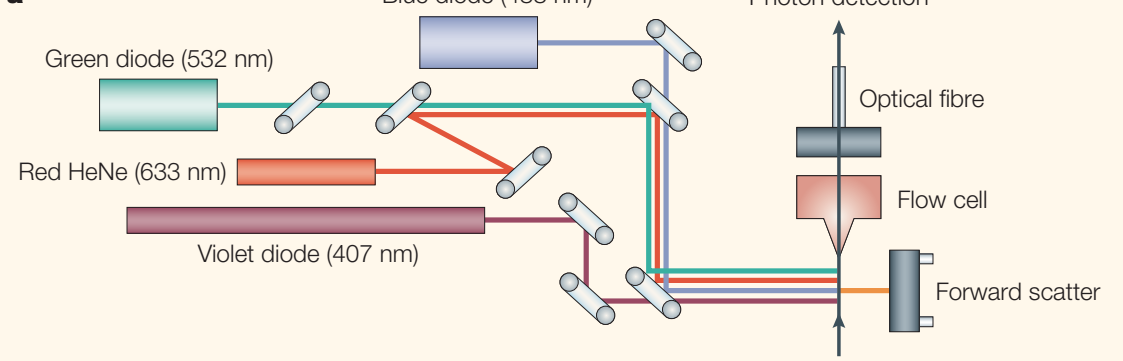

b

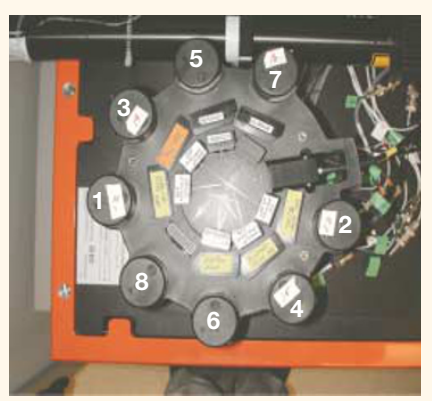

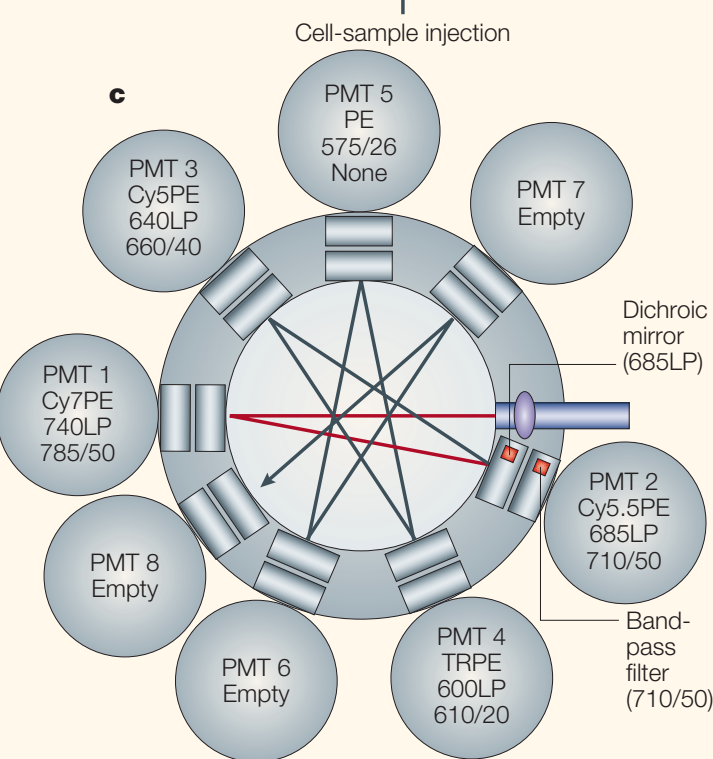

Figure 1 | The laser geometry and fluorescence-emission detection system of a 19-parameter flow cytometer. a $\mid$ A schematic representation shows that this instrument is equipped with three diode-pumped solid-state lasers - blue (488 nm), green (534 nm) and violet (407 nm) - and a red helium-neon ( $\mathrm{HeNe}$ ) laser (633 nm). Each laser line can be independently adjusted through a series of steering mirrors. The lasers are set in the following delay timing order: blue, violet, red then green, with the blue laser as the reference laser. $\mathbf{b} \mid$ A photograph of the octagon assembly for detection of greenlaser-excited emissions is shown. $\mathbf{c} \mid$ A graphical representation of this assembly shows that it is a unique collection system, composed of a series of photomultiplier tubes (PMTs 1-8) and optics in an octagonal arrangement. Photons enter this system and are systematically reflected to the next detector by a dichroic mirror or transmitted through a band-pass filter and counted in that PMT. Dichroic-filter characteristics are shown: for example, for PMT 1, '740LP' (740 nm long-pass filter) indicates that all wavelengths longer than $740 \mathrm{~nm}$ are transmitted and that all wavelengths shorter than $740 \mathrm{~nm}$ are reflected. Band-pass filter characteristics are also shown: for example, for PMT 1, '785/50' indicates that wavelengths in a 50-nm-wide band, which is centred at $785 \mathrm{~nm}$, are transmitted. Each detector quantifies light that is mainly, but not solely, emitted by a single fluorochrome. Cy5PE, cyanine-5-PE; PE, phycoerythrin; TRPE, Texas Red-PE.

differentiation (such as CD45RA, CD27, CD28, CD62L and CC-chemokine receptor 7, CCR7) are expressed on many types of T cell. We still do not understand the redundancy (situations in which multiple phenotypes describe the same cell type) and the gaps (situations in which a distinct cell-surface combination is not ascribed to a functionally unique subset) within and between existing schemes. Do CD45RA-CCR7 + 'central memory' cells exclusively express other, supposedly analogous markers, such as CD62L or CD27? Does the central memory compartment (those cells proposed to provide a durable anamnestic response) contain functionally distinct subsets of cells? These questions can only be answered by adding markers to the simple immunophenotyping schemes that are currently in use. Furthermore, additional markers might be required to study the antigen specificity of T cells (for example, MHCtetramer analysis, in which multimers of peptide-MHC complexes are used to identify $\mathrm{T}$ cells that are specific for a particular antigen), or to study their function (for example, intracellular-cytokine analysis) or other characteristics relevant to particular diseases or patient populations. So, the need for technology that incorporates more than six colours is clear.
The use of additional colours also has distinct advantages for improving the accuracy of measurements of rare cell populations. Specifically, irrelevant cell populations can be excluded from analysis using a 'dump channel', which contains all of the antibodies specific for unwanted cells in one fluorescence channel, such as the Cy5PE (cyanine-5phycoerythrin) channel. Cy5PE is a useful channel for 'dump' antibodies, because dyes that selectively label dead cells, such as propidium iodide and 7AAD, fluoresce in this channel; therefore, any fluorescence in this channel can be used to eliminate dead or unwanted cells. It should be noted that the differential compensation required by the viability dyes and Cy5PE is not important, because all cells that fluoresce in this channel will be eliminated from further analysis. Studies of T-cell populations could include a dump channel for $\mathrm{CD} 19^{+}$cells (B cells), CD56 ${ }^{+}$ cells (NK cells) and CD14 ${ }^{+}$cells (monocytes), with all of the antibodies conjugated to just one fluorochrome. For better detection and discrimination of low-frequency populations, markers shared between the cells of interest and irrelevant cells could be assigned to additional fluorochromes. So, using more colours in an experiment allows moreaccurate and more-sensitive measurements of low-frequency cell populations. This benefit is particularly important in vaccine development, in which the cells that provide immune protection can comprise a small proportion of the overall T-cell compartment.

Over time, the utility of polychromatic flow cytometry is also likely to become apparent in other settings, such as oncology ${ }^{13}$ and haematology. Because the requirements for developing and implementing this technology have now been defined, many of the main hurdles to its widespread adoption have been cleared. This article describes the sophisticated instrumentation, computer algorithms, and advances in reagent technology that now allow use of a 17-colour system.

\section{Developing 17-colour cytometry}

Ten-parameter instrumentation, which measures eight fluorescent colours and two physical parameters, was first described in 1997. Subsequently, the development of a 13-parameter (11-colour) flow cytometer was reported in 2001 (REFS 4,14-16). Although these instruments provided considerable advances over previous generations of cytometers, they needed high-power lasers, were extensively customized and required highly skilled operators. Recently, however, numerous technological and computing advances have produced a new generation 
of instruments that are cheaper, require less technical skill to operate and are adaptable for applications that require as many as 19 parameters. LSR II flow cytometer (Becton, Dickinson and Company Biosciences, San Jose, United States). As illustrated in FIG. 1a, our 19parameter system, the LSR II, uses three solid-state lasers, which emit in the green $(532 \mathrm{~nm})$, blue $(488 \mathrm{~nm})$ and violet $(407 \mathrm{~nm})$ ranges. In addition, a fourth (helium-neon) laser emits in the red $(633 \mathrm{~nm})$ range. These four lasers provide the excitation for the
An example of this technology is the

fluorochrome-conjugated antibodies. After excitation, each fluorochrome emits photons of light with a unique spectrum; this emission is collected in an octagon or trigon detection system specific to each laser. The octagon detection system for the green-laser-excited fluorochomes (that is, phycoerythrin (PE), Texas Red-PE (TRPE), Cy5PE, Cy5.5PE and Cy7PE) is shown in FIG. 1b,c. At each corner of the assembly, a photomultiplier tube detects light in a particular wavelength range, as controlled by the filters in the centre of the assembly; in this way, each detector measures mainly (but not solely) the emission from a particular

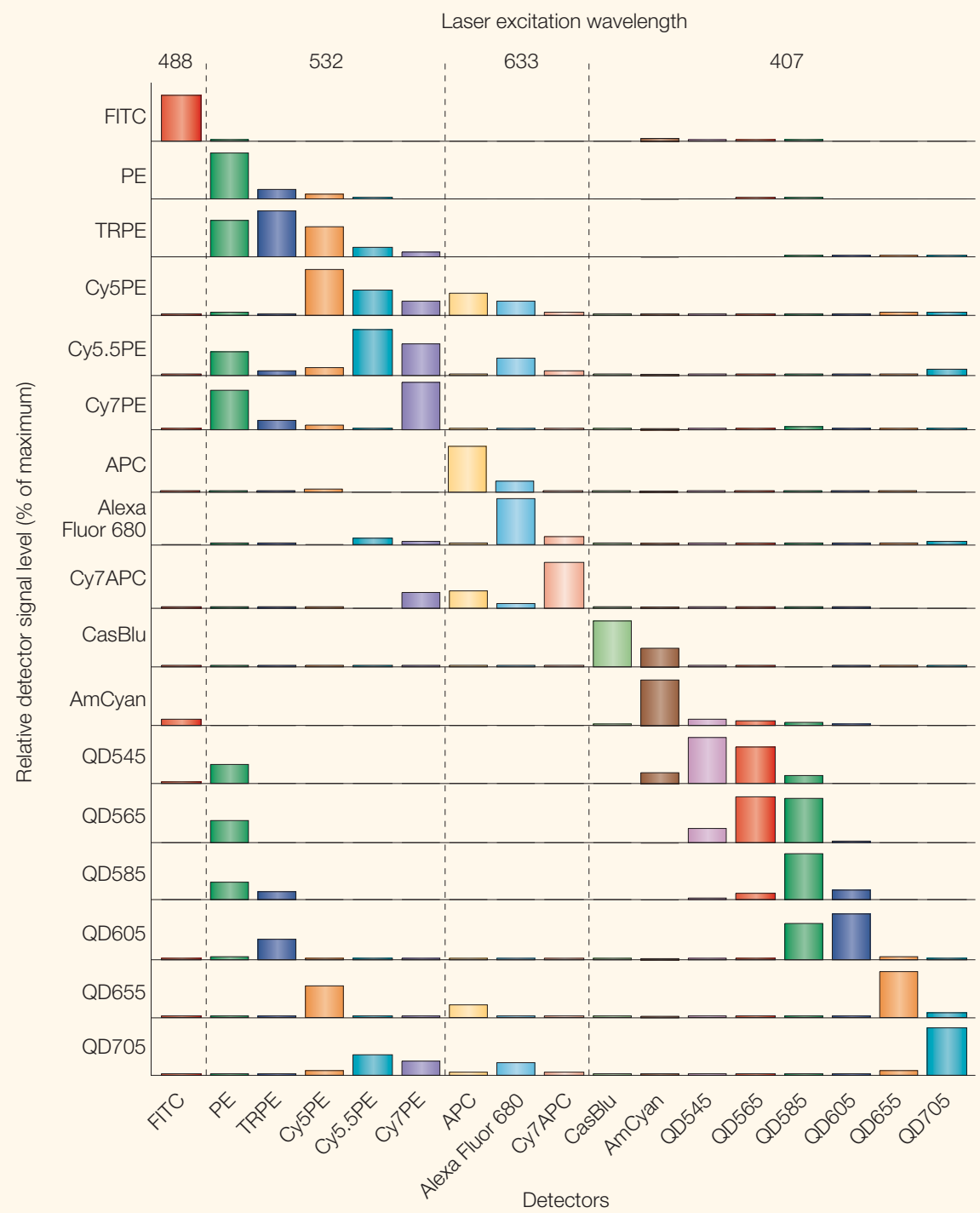

Figure 2 | Spillover from primary fluorochromes into all detectors. Beads were labelled individually with each of 17 fluorochrome-antibody conjugates and analysed on our flow cytometer. The percentage of light from the excitation of the primary fluorochrome that contaminated each of the other detectors was calculated. Each bar represents the percentage of signal in a given detector from a fluorochrome compared to the signal of the fluorochrome in its respective primary detector, so the highest bar in each row represents 100\%. As an example, beads that were stained with Texas Red-phycoerythrin (TRPE) conjugated to CD8-specific antibodies and excited by the blue laser show the highest spillover into the phycoerythrin (PE), cyanine-5-PE (Cy5PE) and Cy5.5PE channels, whereas the emission of Cascade Blue (CasBlu) has little or no spillover into other channels. APC, allophycocyanin; Cy7APC, cyanine-7-APC; FITC, fluorescein isothiocyanate; $\mathrm{QD}$, quantum dot. fluorochrome. This process occurs in two steps. First, the emitted photons encounter one or more dichroic mirrors, which either reflect or transmit photons, depending on the wavelength characteristic of the filter. In this example (FIG. 1c, red line), a 685 long-pass (685LP) dichroic filter reflects wavelengths shorter than $685 \mathrm{~nm}$ and transmits photons with wavelengths longer than $685 \mathrm{~nm}$. Then, the transmitted light is further 'purified' by a band-pass filter before it reaches the detector (using, for example, a 710/50 band-pass filter, which eliminates photons outside the range 685-735 nm). So, the signature excitation and emission curve of each fluorochrome produces photons that are selectively measured by a series of dichroic mirrors and band-pass filters. The main objective of this system is to have the narrowest filter range possible, while still capturing the maximum amount of light. In the 17-colour detection system that we built, the green laser is configured with five detectors (for the fluorochromes PE, TRPE, Cy5PE, Cy5.5PE and Cy7PE). If additional fluorochromes become available that have distinct emission spectra after excitation by the green laser, the detection capacity could be increased even further. The blue laser is configured with a single fluorescence detector (for fluorescein isothiocyanate, FITC); the red laser is configured with three detectors (for allophycocyanin (APC), Cy7APC and Alexa Fluor 680; and the violet laser is configured with eight detectors (for quantum dots (also known as Q dots or QDs) -545, QD565, QD585, QD605, QD655, QD705, AmCyan and Cascade Blue). As more fluorescent dyes become available, the detection capacity can be increased through the incorporation of additional lasers or more-complex detection assemblies; there is no theoretical limit on the number of fluorochromes that can be simultaneously measured.

\section{Developing complex reagent panels}

The development of reagent panels for immunophenotyping begins with the selection of surface or intracellular proteins for study. Ideal markers for immunophenotyping are proteins that are likely to be relevant in the life cycle of a cell type, such as the lymphnode homing molecules CD62L and CCR7 for naive $\mathrm{T}$ cells ${ }^{17-20}$. In addition, the ideal marker (or combination of markers) would be differentially expressed across the cell subsets to be investigated. Finally, the best markers for phenotyping cell subsets are unaltered in three situations: first, in vitro stimulation, which is required for many functional assays; second, viral infection, which might alter 
expression of cell proteins as an immuneevasion mechanism; and third, cell cryopreservation, which can, for example, strip CD62L from the membrane of frozen cells. The latter is a particularly important consideration for immunogenicity analysis in large-scale vaccine trials, in which it is untenable to carry out all assays on freshly isolated cells.

After antibodies specific for the markers of interest have been selected, the most suitable fluorochromes for detection need to be chosen. The availability of many fluorescent dyes, with distinct excitation or emission spectra, allows for several different fluorochrome-antibody pairings, which can be combined to produce a large number of possible staining panels. For example, in an 11colour flow-cytometry experiment, there are nearly 40 million different staining panels that can be assembled by assigning antibodies to different fluorochromes, and for an 11colour experiment with 17 available channels, there are more than $5 \times 10^{11}$ possibilities. Although the best combination of antibodies and fluorochromes for a particular application can as yet only be ascertained empirically, there are considerations that can guide users in their design of staining panels.

The assignment of antibodies to fluorochromes is determined mainly by the background staining of, and spectral overlap between, the chosen fluorescent dyes. Some background staining is generated by the natural autofluorescence of organic molecules in cells (such as NADH); the emission spectrum of autofluorescence overlaps that of dyes such as Cascade Blue, FITC and PE. High background staining can also result from the nonspecific binding of a fluorescent conjugate to cellular elements, which occurs when the antibody concentration is too high. In either case, the problems caused by high background staining can be avoided by the titration of antibody reagents before their use. From titration data, the antibody concentration that provides the maximal brightness of the positive population with the least amount of background staining should be chosen for use in the panel' ${ }^{21}$.

The spectral overlap between fluorescent dyes also needs to be considered in the assignment of antibodies to fluorochome channels. This overlap needs to be mathematically eliminated using a process known as compensation. Compensation is the mathematical deconvolution of the outputs from multiple detectors to yield measurement values proportional to each of the fluorescent dyes used. The need for compensation arises from the spillover of light between detectors
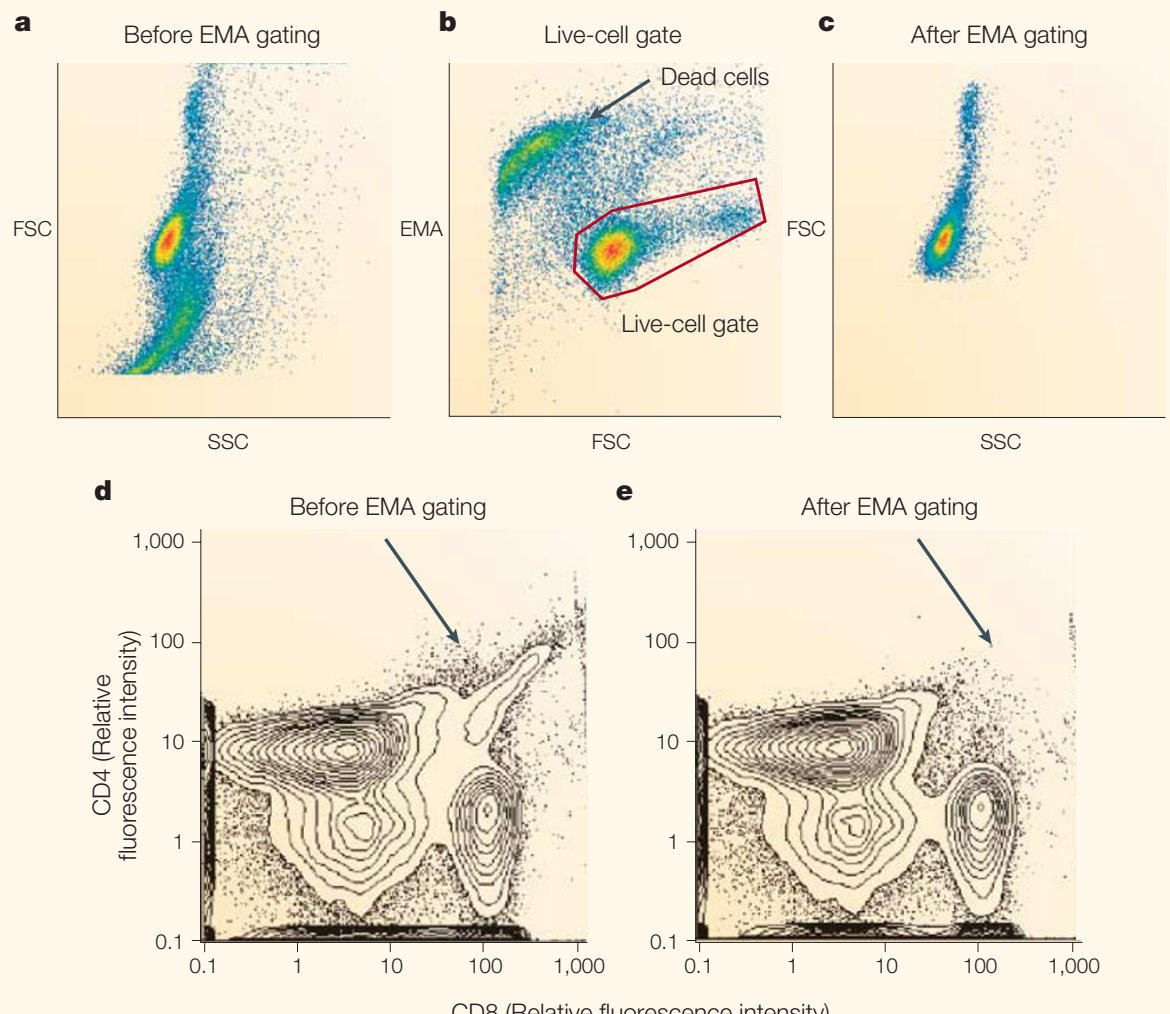

CD8 (Relative fluorescence intensity)

Figure 3 | Dead cells can be an important artefact. In this sample experiment, numerous dead cells are apparent in the light-scatter gate (a); however, not all of these can be effectively removed solely by gating on forward scatter (FSC) and side scatter (SSC). These cells can be effectively removed from the analysis by adding ethidium monoazide (EMA) to the cell sample and then gating on the EMA' cells (live-cell gate) (b). Further analysis uses the light-scatter gate after this 'EMA gating' (c). Data are shown for cells stained with CD4- and CD8-specific antibodies before (d) and after (e) EMA gating. A doublepositive population (arrow) can be observed as a result of the dead cells present in the light-scatter gate. Until now, the identification of dead cells has not been crucical in many experiments. However, because polychromatic flow cytometry can identify hundreds of cell subsets, many of these will be present at frequencies below the fraction of dead cells (typically a few percent of all cells). Therefore, it is crucial to eliminate even this small percentage of dead cells from the analysis, to ensure that they do not artefactually comprise most of the subset of interest.

and the relatively wide excitation energies of most fluorochromes. As illustrated in FIG. 2, signals emitted by one fluorochrome ( $y$ axis) can contaminate neighbouring detectors ( $x$ axis), which results in 16 potential sources of contaminating light. For example, the TRPE fluorochrome shows a large percentage spillover into the PE, Cy5PE and Cy5.5PE detectors. By contrast, light from FITC and Cascade Blue have little spillover into other detectors.

However, even with computer-assisted compensation, inescapable measurement errors lead to a broadening of the distribution of the fluorescence of cells that have low or no fluorescence, particularly in the far-red channels (APC, Alexa Fluor 680 and Cy7APC) ${ }^{14,22}$. This phenomenon can adversely affect the resolution between dimly staining cell populations and negative cell populations (that is, populations for which there is no detectable cell-surface expression of the marker). So, markers that stain brightly and provide good separation between positive and negative cell populations should be chosen for the far-red channels (for example, antibodies specific for CD3, CD4 and CD8). Bright fluorochromes, such as PE or Cy5PE, should be used for markers with unknown staining patterns or for markers that require clear discrimination between dimly staining and negative cell populations (for example, CD28 and CD127). The latter types of marker might also be tested with fluorochromes that have little spillover from other channels, such as those excited by $407 \mathrm{~nm}$ violet lasers (for example, Cascade Blue).

Recently, new reagents were introduced to expand the violet-laser excitation repertoire. QDs are composed of cadmium and selenium molecules of $10-15 \mathrm{~nm}$, which are covered by an outer shell of zinc sulphate. These particles are coated with polymers, thereby allowing attachment of monoclonal 


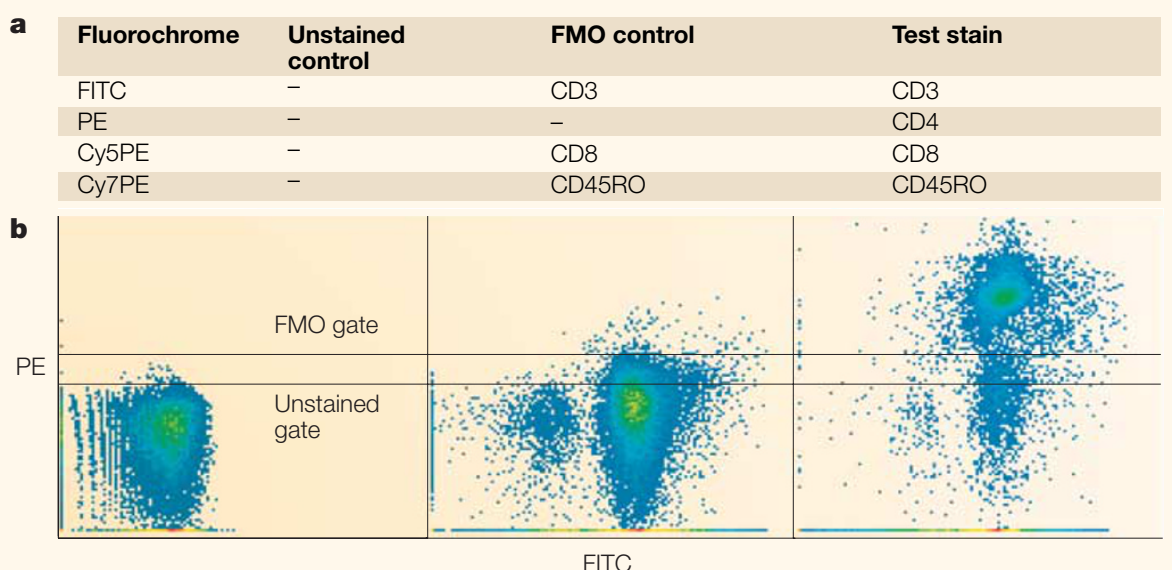

Figure 4 | Use of FMO controls. a | In this sample experiment, a fluorescence-minus-one (FMO) control was devised to optimize the identification of $\mathrm{CD}^{+}$and $\mathrm{CD} 4^{-} \mathrm{T}$ cells. $\mathbf{b}$ | The unstained control, the FMO control and the fully stained cell sample (test stain) - stained with anti-CD3-FITC (fluorescein isothiocyanate), anti-CD4-PE (phycoerythrin), anti-CD8-Cy5PE (cyanine-5-PE) and anti-CD45RO-Cy7PE - are shown after compensation. The FMO control is a better indicator of negative-control position ('FMO gate' line) than the unstained control ('unstained gate' line). It should be noted that the 'FMO gate' line is the one-dimensional FMO gate applied to the CD4 channel. A better FMO gate would be a curved line (REF. 22); the ideal FMO gate would be a four-dimensional surface separating expressing from non-expressing cells.

antibodies or streptavidin. By varying the amount of cadmium and selenium, QDs can emit light in a broad range (525-705 $\mathrm{nm}$ ); therefore, after conjugation to monoclonal antibodies, these reagents increase the colour capabilities of the $407 \mathrm{~nm}$ violetlaser detection system from two to eight parameters $^{23}$. Another recent addition to the violet-laser repertoire is the organic fluorochrome AmCyan. This reagent is brighter than older reagents that are detected in the violet laser channel, such as Alexa Fluor 430. Both QD and AmCyan are attractive choices for low-power lasers, such as the violet solid-state laser.

Although these new reagents make it possible to envisage 17-colour panels, new studies need to begin with preliminary experiments to assess the staining patterns of smaller panels that use four or five well-defined markers. The staining patterns observed in these smaller panels then need to be compared with those previously reported or previously observed in simple two- or three-colour experiments. If the results are not comparable, then new combinations of fluorochromes need to be tested. After comparable results are obtained, the investigator could add markers of interest to the panel, but care should be taken to ensure that the staining patterns of the original markers are not changed by the addition of new markers.

Two crucial considerations in the development of new staining panels are the use of viability markers to exclude dead cells and the incorporation of controls to help identify cell subsets based on their fluorescence measurements. Viability markers are often required in experiments in which the cells of interest are rare (less than $1 \%$ of the total population) or have been activated or thawed. In these types of sample, cell death can result in large errors because of the propensity of dead cells to bind nonspecifically to monoclonal antibody conjugates. For example, FIG. 3 shows how the viability marker ethidium monoazide (EMA) can eliminate a substantial number of dead cells from the light-scatter dot plot (FIG. 3a), allowing more-precise identification of lymphocytes (FIG. 3c). In FIG. 3d, the way in which staining artefacts, such as the double-positive $\left(\mathrm{CD} 4^{+} \mathrm{CD}^{+}\right)$staining of peripheral blood cells (indicated by the arrow), can be attributed to dead cells in the lymphocyte gate is shown. It should be noted that these doublepositive cells are not present in the sample after the $\mathrm{EMA}^{+}$cells have been gated out (FIG. 3e).

The second important aspect to panel design is the inclusion of a 'fluorescenceminus-one' (FMO) control. This is a sample that has been stained with all of the reagents except one; the analysis of this sample allows for the precise definition of cells that have fluorescence above background levels (FIG. 4). In this example, cells stained with anti-CD3-FITC (that is, FITCconjugated CD3-specific monoclonal antibodies), anti-CD4-PE, anti-CD8-Cy5PE and anti-CD45RO-Cy7PE are compared with cells stained with all monoclonal-antibody conjugates except the CD4-specific antibodies (FMO control) (FIG. 4a). It should be noted that by using the unstained sample to determine the cut-off between positive and negative cell populations ('unstained gate' line), the population gate would be too low to accurately distinguish $\mathrm{CD}^{+}$and $\mathrm{CD} 4^{-} \mathrm{T}$ cells (FIG. 4b). However, the cut-off determined by the FMO control ('FMO gate' line) can correctly separate these populations, thereby giving a more accurate representation of the percentage of the population that comprises $\mathrm{CD} 4^{+}$cells $^{21}$.

\section{Analysis tools for 19-parameter data}

Data analysis is by far the most timeconsuming aspect of polychromatic flowcytometry experiments; therefore, there is considerable demand for tools that can organize analyses into databases, as well as assist in the exploration of complex data sets.

Recently, automated data-analysis techniques were developed to identify subpopulations of cells in complex multivariate data. These automated techniques are intended to complement the commonly used technique of dividing data by manually segregating events into regions two parameters at a time (known as bivariate gating). The automated approach is similar to cluster algorithms, which group individual datum points together based on similarity or disparity; the computation of similarity is the core of such algorithms and varies markedly between different approaches. The algorithms are based on published techniques that quantify the difference between multivariate distributions; these are known as probability-binning comparison algorithms ${ }^{24-26}$. Automated subset-identification algorithms might also be useful in settings in which cells respond in complex, multivariate patterns or for the rapid identification of immunophenotyping patterns that correlate with pathogenic states $^{27}$.

Examples of cluster- and bivariate-gating analyses are shown in FIG. 5. The antibodies that were used for staining are indicated in the figure. It should be noted that cluster analysis identifies many different cell populations and assigns a different colour to each figure (FIG. 5a). The expression level of each marker can then be visualized for the various clusters by using dot plots and histograms. So, the orange cluster mainly contains $\mathrm{CD} 4^{+} \mathrm{T}$ cells (arrow 1), whereas the green and yellow clusters are mainly $\mathrm{CD}^{+} \mathrm{T}$ cells (arrow 2 ). The main advantage of this analysis is the ability to rapidly (and automatically) explore populations without the constraints of defined gates. For example, cluster analysis can quickly identify a population of $\mathrm{CD} 4^{+} \mathrm{CD} 45 \mathrm{RO}^{+} \mathrm{T}$ cells that express both interleukin-2 (IL-2) (arrow 3) and interferon- $\gamma($ IFN- $\gamma$ ) (arrow 4). Similarly, 
a Automated cluster analysis
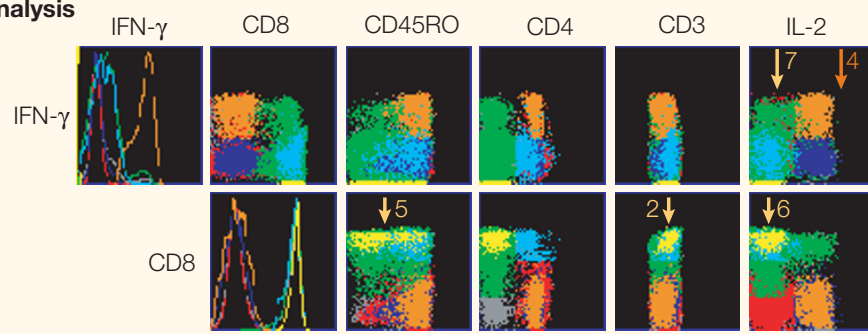

CD8
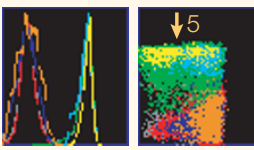

b Manual bivariate-gating analysis
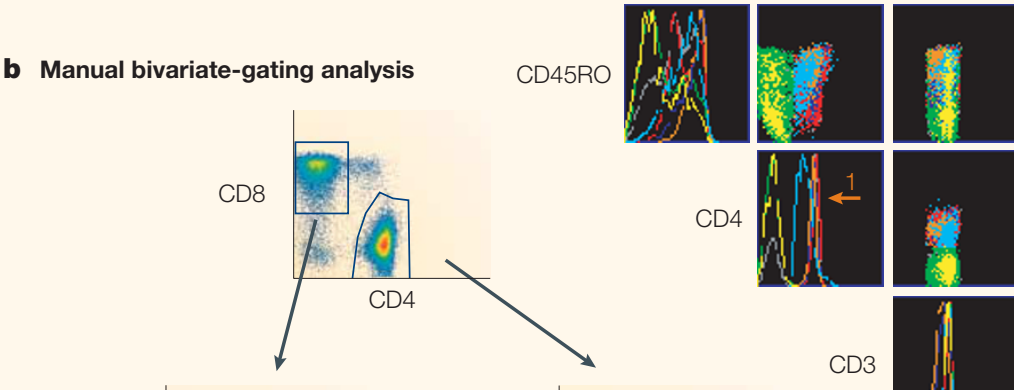

CD4
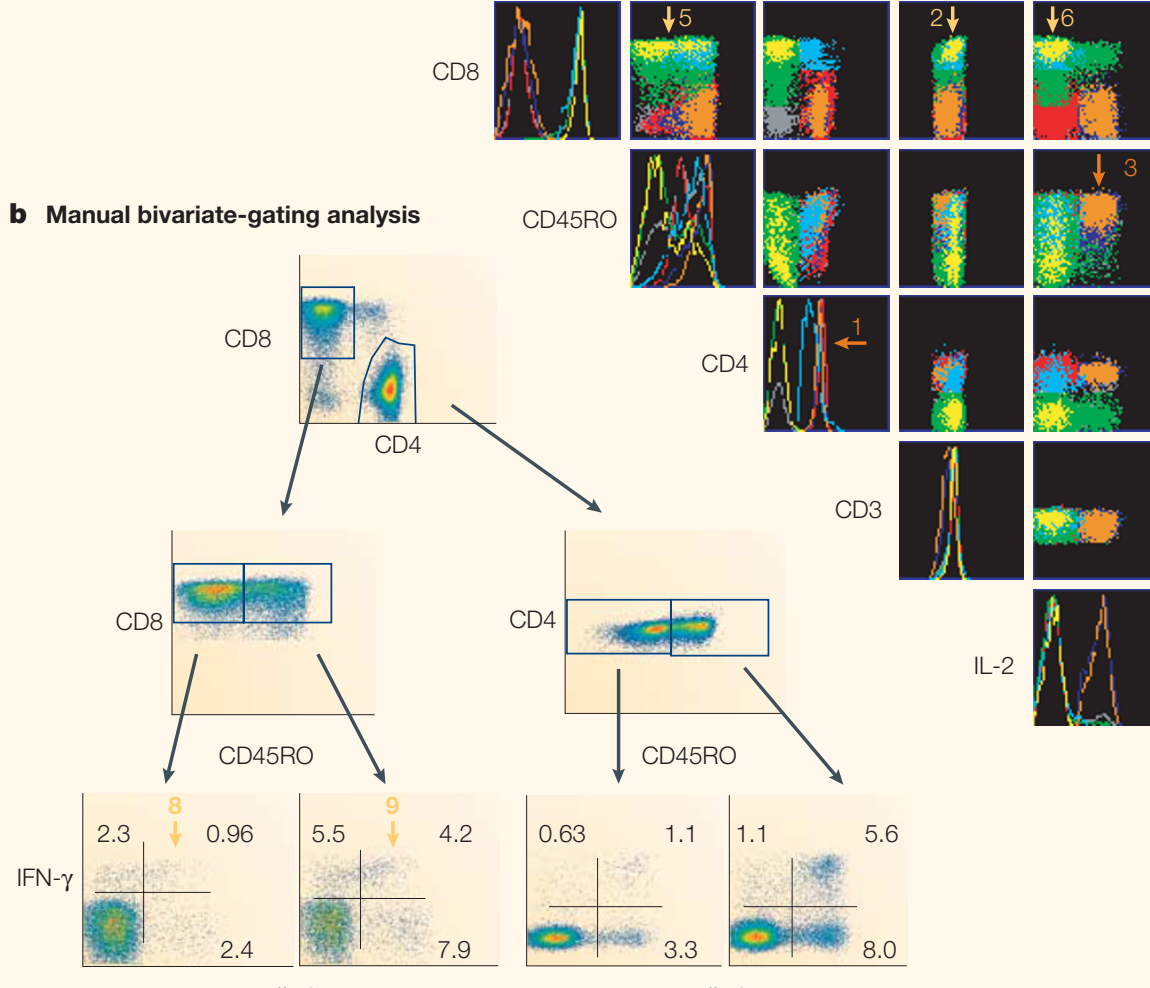

IL-2

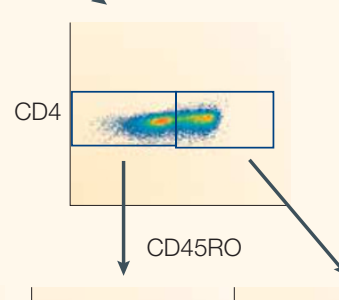

CD3
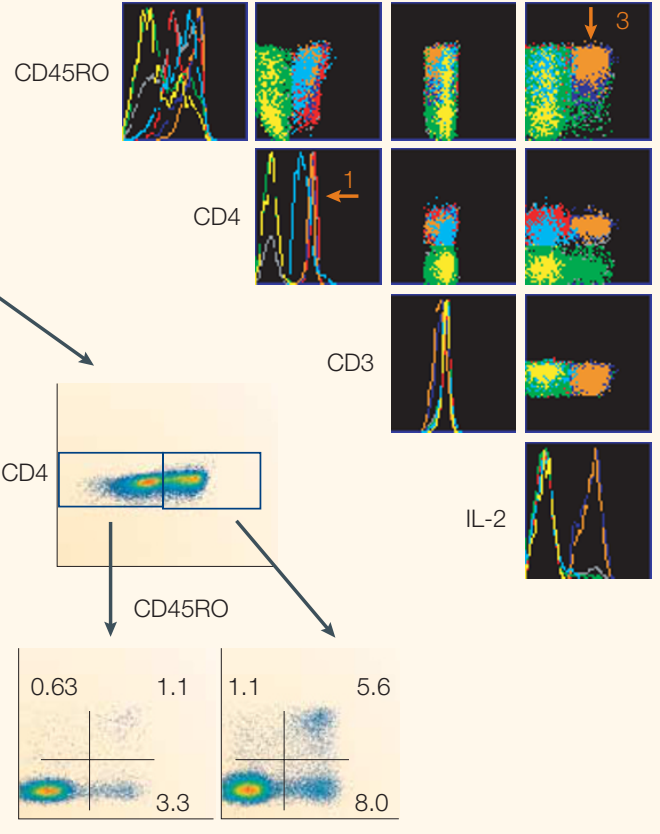

IL-2

Figure 5 | Comparison of automated cluster analysis and manual bivariate analysis. Peripheralblood mononuclear cells were stimulated with staphylococcal enterotoxin B (for 6 hours in the presence of brefeldin A) then permeabilized and stained with antibodies specific for various cell-surface markers and intracellular cytokines. Data are gated on $\mathrm{CD}^{+} \mathrm{T}$ cells. a | Cluster analysis identifies multiple cell populations, each of which is assigned a particular colour. In this example, orange clusters consist of $\mathrm{CD}^{+} \mathrm{T}$ cells (arrow 1), whereas green and yellow clusters consist of CD8 ${ }^{+} \mathrm{T}$ cells (arrow 2). Cluster analysis can quickly identify CD4+ $\mathrm{T}$ cells that express CD45RO and interleukin-2 (IL-2) (arrow 3), together with interferon- $\gamma(\mathrm{IFN}-\gamma$ ) (arrow 4). Similarly, heterogeneous expression of CD45RO (arrow 5, yellow population) is quickly recognized in CD8+ T cells that lack IL-2 expression (arrow 6). So, automatic identification and visualization of clusters can be a powerful tool. However, because overlaid clusters can be difficult to distinguish, the level of IFN- $\gamma$ expression in the CD8+IL-2- population is unclear (that is, the yellow population under arrow 7 cannot be visualized). b | Manual bivariate gating resolves this problem, because the level of IFN- $\gamma$ expression can be visualized and, more importantly, quantified for populations with varying expression levels of CD8, CD45RO and IL-2 (arrows 8 and 9). The numbers indicate the percentage of cells present in each quadrant.

a CD8 ${ }^{+} \mathrm{IL}^{-2} 2^{-}$cell population (arrow 6 ) that is heterogeneous in its expression of CD45RO (arrow 5) is also readily apparent. However, in this analysis, because the overlaid colours in the cluster analysis can be difficult to distinguish, the level of IFN- $\gamma$ expression in the $\mathrm{CD} 8^{+} \mathrm{IL}-2^{-}$cell population is unclear (arrow 7).

When bivariate-gating analysis is used (FIG. 5b), clearer more-quantitative results are obtained, albeit at the expense of considerable analyst time. For instance, bivariate analysis shows the presence of $\mathrm{CD}^{+} \mathrm{IFN}-\gamma^{+}$ cells that do not express IL-2. Moreover, the relationship between cytokine production by $\mathrm{CD}^{+} \mathrm{CD}^{2} 5 \mathrm{RO}^{-}$cells (arrow 8 ) and $\mathrm{CD}^{+} \mathrm{CD} 45 \mathrm{RO}^{+}$cells (arrow 9) can be directly assessed. To summarize, cluster analysis automatically provides a visual display that enables rapid determination of the level of expression of multiple markers across multiple dimensions; however, it may not provide the clear, structured and quantitative analysis that bivariate gating does. At present, cluster analysis is recommended as a tool for rapid, qualitative (not quantitative) data exploration. Certainly, more tools that aid data exploration are needed.
As indicated earlier, the multidimensional nature of data from polychromatic experiments can make cluster analyses unwieldy. Therefore, there is a need for other visualization tools that simultaneously allow data exploration, comparison and quantification with maximum flexibility. In this context, flexibility indicates the ability to rapidly rearrange the way in which data are presented, by choosing a new parent phenotype to anchor the analysis or by hiding certain markers. Representing data in a form similar to a hierarchical tree, such as the one presented in FIG. 6, might offer some of these advantages. In this type of graphical representation, each marker that is measured (such as CD11a) is assigned to a particular level of the tree, and different branches represent various expression levels of the marker (for example, positive versus negative) in the immediate parent population. Phenotypes that do not occur in a cell population are not displayed on the tree, allowing rapid comparison of samples according to the 'shape' of the tree. To further explore the data, the order of the markers can be rearranged until branches with similar functional characteristics are brought together, thereby identifying phenotypes that are similar in function. For example, high IFN- $\gamma$ production is shown to be confined to CD11 $\mathrm{a}^{\text {bright }}$ cells in FIG. 6 . Similarly, the overlap between the markers that are examined could be determined by comparing the full phenotypic tree to a tree in which one or more markers are hidden. Finally, as indicated in FIG. 6, quantitative elements could be added to tree graphics. In the simplest case, a frequency histogram underneath the tree can show the proportion of cells that belong to a particular branch. Alternatively, the histogram could indicate the frequency of a subset that shows a certain function. Such tree-based graphics offer many options for data exploration, comparison and quantification.

\section{Concluding remarks}

As immunologists further dissect the heterogeneity of the cell populations that coordinate defences against various pathogens, polychromatic flow cytometry will be an invaluable tool. Until recently, it has been limited by instrument complexity, reagent availability and a lack of powerful analysis software; however, all of these areas are now progressing, thereby enabling this technology to advance rapidly.

Nonetheless, there remain considerable hurdles before this application becomes widely used. First, we need flow cytometers 


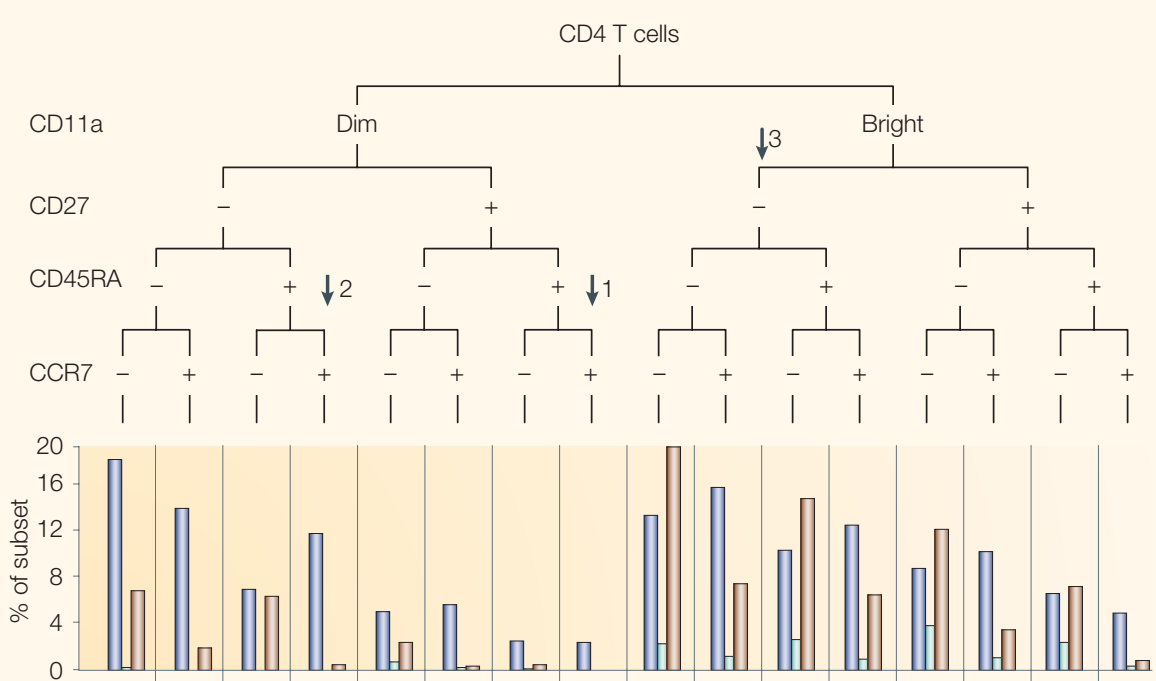

$\square \mathrm{IL}-2^{+} \mathrm{IFN}-\gamma^{-} \square \mathrm{IL}-2^{-} \mathrm{IFN}-\gamma^{+} \square \mathrm{IL}-2^{+} \mathrm{IFN}-\gamma^{+}$

Figure 6 | Visualization of polychromatic data using hierarchical trees. The data shown in this hierarchical tree were obtained from peripheral-blood mononuclear cells stimulated with staphylococcal enterotoxin B (for 6 hours in the presence of brefeldin A). The cells were subsequently permeabilized and stained for various cell-surface markers and intracellular cytokines. Branches of the tree segregate based on the expression level (for example, positive versus negative or bright versus dim) of a particular marker, and each terminal branch represents one fully gated subset. So, reading from the top, the branch below arrow 1 represents cells that are $C D 3{ }^{+} C D 4{ }^{+} C D 11 a^{\text {dim }} C D 27{ }^{+} C D 45 R A{ }^{+} C C$-chemokine receptor $7(\mathrm{CCR} 7)^{+}$. The frequency histogram below the tree indicates the relative proportion of cells in each subset that express interleukin-2 (IL-2) (blue bars), interferon- $\gamma$ (IFN- $\gamma$ ) (green bars) or both (red bars). So, the CD11 $a^{\text {dim }} \mathrm{CD} 27^{+} \mathrm{CD} 45 \mathrm{RA}{ }^{+} \mathrm{CCR} 7^{+}$cell population indicated by arrow 1 expresses only IL-2, which is consistent with a naive phenotype. Interestingly, IL-2 is expressed by a greater fraction of a naive-like subset (CD11a dim CD27-CD45RA+CCR7 ${ }^{+}$cells), in which cells have lost expression of CD27 but not other markers (arrow 2). In contrast, high expression of IFN- $\gamma$ is mainly observed in CD11a ${ }^{\text {bright }} C D 27^{-}$cells (arrow 3), a phenotype previously associated with effector memory $T$ cells. Graphical representations such as this offer many options for data exploration, especially when the hierarchy can be rearranged to bring patterns into view.

that are easier to validate and have consistent ways of implementing lasers and opticaldetection systems (filter combinations) so that each configuration need not be separately optimized. Second, more-sophisticated systems are needed for software-controlled data acquisition, to help guide the user through the still relatively complex set-up and optimization procedure. Third, manufacturers need to devise a new model for reagent distribution so that laboratories can test numerous conjugated monoclonal antibodies at minimal cost before committing to purchase larger amounts of the final optimized panels. This will require manufacturers to have a large inventory, because the number of reagent-fluorochrome combinations will be an order of magnitude greater than what is required at present. Last, we desperately need new tools to aid in the exploration of these complex data sets, as well as new techniques for the presentation of data. Journals will need to accommodate the requirement for the presentation of farmore complex graphics and analyses, and the inclusion of such information as online supporting documentation will become ubiquitous.

Undoubtedly, polychromatic flowcytometry technology such as we describe here will become more common in immunology and clinical-research laboratories worldwide. In our laboratory, we use this technology to identify correlates of immune protection, therapeutic efficacy and disease morbidity for disease caused by HIV and for vaccination against infectious diseases. Although the identification of such correlates might not require more than six- to eightcolour flow cytometry, the process will be markedly accelerated by the ability to make many more measurements simultaneously.

Stephen P. Perfetto, Pratip K. Chattopadhyay and Mario Roederer are in the ImmunoTechnology Section, Vaccine Research Center, National Institute of Allergy and Infectious Disease, National

Institutes of Health, 40 Convent Drive, Room 5507, Bethesda, Maryland 20892-3015, United States. e-mail:sperfetto@nih.gov

doi:10.1038/nri1416 Correspondence to S.P.P.
1. Bonner, W. A., Hulett, H. R., Sweet, R. G. \& Herzenberg L. A. Fluorescence activated cell sorting. Rev. Sci. Instrum. 43, 404-409 (1972).

2. Herzenberg, L. A. \& Sweet, R. G. Fluorescence-activated cell sorting. Sci. Am. 234, 108-117 (1976).

3. Hulett, H. R., Bonner, W. A., Barrett, J. \& Herzenberg, L. A. Cell sorting: automated separation of mammalian cells as a function of intracellular fluorescence. Science $\mathbf{1 6 6}$ 747-749 (1969).

4. De Rosa, S. C., Herzenberg, L. A. \& Roederer, M. 11 color, 13-parameter flow cytometry: identification of human naive $T$ cells by phenotype, function, and T-cell receptor diversity. Nature Med. 7, 245-248 (2001).

5. De Rosa, S. C. \& Roederer, M. Eleven-color flow cytometry. A powerful tool for elucidation of the complex immune system. Clin. Lab. Med. 21, 697-712 (2001).

6. Roederer, M., Dubs, J. G., Anderson, M. T., Raju, P. A. \& Herzenberg, L. A. CD8 naive T cell counts decrease progressively in HIV-infected adults. J. Clin. Invest. $\mathbf{9 5}$, 2061-2066 (1995).

7. Rabin, R. L., Roederer, M., Maldonado, Y., Petru, A. \& Herzenberg, L. A. Altered representation of naive and memory CD8 T cell subsets in HIV-infected children. J. Clin. Invest. 95, 2054-2060 (1995).

8. Hatzakis, A. et al. Effect of recent thymic emigrants on progression of HIV-1 disease. Lancet 355, 599-604 (2000).

9. Schnittman, S. M. et al. Preferential infection of $\mathrm{CD}^{+}$ memory $T$ cells by human immunodeficiency virus type 1 : evidence for a role in the selective T-cell functional defects observed in infected individuals. Proc. Natl Acad. Sci. USA 87, 6058-6062 (1990).

10. Douek, D. C. et al. HIV preferentially infects HIV-specific $\mathrm{CD4}^{+} \mathrm{T}$ cells. Nature 417, 95-98 (2002).

11. Hazenberg. M. D. et al. Persistent immune activation in HIV-1 infection is associated with progression to AIDS. AIDS 17, 1881-1888 (2003).

12. Margolick, J. B. et al. Failure of T-cell homeostasis preceding AIDS in HIV-1 infection. The multicenter AIDS cohort study. Nature Med. 1, 674-680 (1995).

13. Lee, P. P. et al. Characterization of circulating T cells specific for tumor-associated antigens in melanoma patients. Nature Med. 5, 677-685 (1999).

14. Roederer, M. et al. 8 color, 10-parameter flow cytometry to elucidate complex leukocyte heterogeneity. Cytometry 29, 328-339 (1997).

15. De Rosa, S. C., Brenchley, J. M. \& Roederer, M. Beyond six colors: a new era in flow cytometry. Nature Med. 9, 112-117 (2003).

16. Herzenberg, L. A., Parks, D., Sahaf, B., Perez, O. \& Roederer, M. The history and future of the fluorescence activated cell sorter and flow cytometry: a view from Stanford. Clin. Chem. 48, 1819-1827 (2002).

17. Brenchley, J. M. et al. Expression of CD57 defines replicative senescence and antigen-induced apoptotic death of CD8 ${ }^{+}$T cells. Blood 101, 2711-2720 (2003).

18. Champagne, P. et al. Skewed maturation of memory HIVspecific CD8 T lymphocytes. Nature 410, 106-111 (2001).

19. Sallusto, F., Lenig, D., Forster, R., Lipp, M. \& Lanzavecchia, A. Two subsets of memory T lymphocytes with distinct homing potentials and effector functions. Nature 401, 708-712 (1999).

20. Unsoeld, H., Krautwald, S., Voehringer, D. Kunzendorf, U. \& Pircher, H. CCR7 $7^{+}$and CCR7- memory T cells do not differ in immediate effector cell function. J. Immunol. 169, 638-641 (2002).

21. Perfetto, S., De Rosa, S. D. \& Roederer, M. in Flow Cytometry (eds Darzynkiewicz, D., Tanke, H. \& Roederer, M.) (Elsevier, San Diego, in the press).

22. Roederer, M. Spectral compensation for flow cytometry: visualization artifacts, limitations, and caveats. Cytometry 45, 194-205 (2001).

23. Bruchez, M. Jr, Moronne, M., Gin, P., Weiss, S. \& Alivisatos, A. P. Semiconductor nanocrystals as fluorescent biological labels. Science 281, 2013-2016 (1998).

24. Roederer, M. \& Hardy, R. R. Frequency difference gating: a multivariate method for identifying subsets that differ between samples. Cytometry 45, 56-64 (2001).

25. Roederer, M., Moore, W., Treister, A., Hardy, R. R. \& Herzenberg, L. A. Probability binning comparison: a metric for quantitating multivariate distribution differences. Cytometry 45, 47-55 (2001).

26. Roederer, M., Treister, A., Moore, W. \& Herzenberg, L. A. Probability binning comparison: a metric for quantitating univariate distribution differences. Cytometry 45, 37-46 (2001).

27. Baggerly, K. A. Probability binning and testing agreemen between multivariate immunofluorescence histograms: extending the $\chi^{2}$ test. Cytometry 45, 141-150 (2001). 
Acknowledgements

The development of this technology began in the Herzenberg laboratory at Stanford University (Stanford, United States), and we are grateful for the critical support and enthusiasm that Leonard and Leonore Herzenberg have provided. In addition, we recognize the enormous contributions made to the development of this technology by Stephen De Rosa, while he was in our laboratory. We also thank Joanne Yu and other members of our laboratory for help in reagent development and validation. The National Institutes of Health does not endorse or recommend any commercial products, processes or services. The views and opinions expressed in this manuscript do not necessarily state or reflect those of the United States government, and they may not be used for advertising or product-endorsement purposes. The United States government does not warrant or assume any legal liability or responsibility for the accuracy, completeness or usefulness of any information, apparatus, product or process disclosed.

Competing interests statement

The authors declare competing financial interests: see Web version for details.

\section{(4) Online links}

DATABASES

The following Entrez Gene: http://www.ncbi.nlm.nih.gov/entrez/query.fcgi?db=gene
CCR7 | CD4 | CD8 | CD11a | CD14 | CD19 | CD27 | CD28 CD45 | CD56 | CD62L | CD127 | IFN- $\gamma \mid$ | IL-2

\section{FURTHER INFORMATION}

\section{Mario Roederer's laboratory:}

http://vrc.nih.gov/VRC/labs_immunotechnology.htm

Multicolour flow-cytometry instrumentation Becton, Dickinson and Company Biosciences:

http://www.bd.com/

Beckman Coulter, Inc.:

http://www.beckman.com

DakoCytomation:

http://www.dakocytomation.com/

Partec GmbH:

http://www.partec.de/
Fluorochrome-conjugated antibodies

Becton, Dickinson and Company Biosciences:

http://www.bd.com/

Beckman Coulter Inc.:

http://www.beckman.com

DakoCytomation:

http://www.dakocytomation.com/

Caltag Laboratories:

http://www.caltag.com

Fluorochromes and kits for conjugation

Molecular Probes, Inc.: http://www.probes.com

R\&D Systems: http://www.rndsystems.com

ProZyme: http://www.prozyme.com

Multicolour cytometric data-analysis software

Tree Star, Inc.: http://www.treestar.com

FCSPress: http://www.fcspress.com/

De Novo Software:

http://www.denovosoftware.com/

Verity Software House: http://www.vsh.com/

Access to this interactive links box is free online. 